\title{
Interpretation of rock mass deformability measurements using large flat jack tests
}

\author{
B. Figueiredo, F. Bernardo, L. Lamas \& J. Muralha \\ National Laboratory for Civil Engineering (LNEC), Lisbon, Portugal
}

\begin{abstract}
Large flat jack tests are used to assess rock mass deformability in the design stages of large projects. This test consists in cutting a thin slot in the rock mass in which a flat jack with large dimensions is inserted; oil pressure inside the jack loads the slot walls while the rock mass deformation is measured by displacement gauges located inside the jack and at the surface. The main difficulty regarding interpretation of large flat jack tests has to do with the effect of the tension crack that may develop in the rock mass during the tests. A three-dimensional numerical model was developed for the interpretation of large flat jack test results. The model was used to establish the relations between the in situ stress perpendicular to the slots, the rock mass tensile strength, the applied pressures and the crack depth.
\end{abstract}

SUBJECT: Modeling and numerical methods

KEYWORDS: Field measurements, numerical modeling, rock properties, site characterization

\section{INTRODUCTION}

Rock mass deformability plays a significant role in the design of large structures such as concrete dams, large bridges, underground caverns and tunnels, since their behaviour depends on the displacements undergone by the rock mass. For the design of these types of structures, it is not adequate to characterize rock mass deformability just using laboratory tests on intact rock specimens, and extrapolating their results to the rock mass based on subjective indices such as the RMR, Q or GSI values. In situ tests involving relatively large and representative rock mass volumes can provide reliable estimates of rock mass deformability, and allow reducing uncertainties during design stages.

Large flat jack (LFJ) tests are one of the field techniques used to assess the rock mass deformability that allow testing relatively large volumes of rock mass (Rocha 1974). LFJ tests begin with cutting a thin slot in the rock mass using a disk saw, and inserting a flat jack. Then, hydraulic pressure in the flat jacks applies loads to the slot walls, while rock mass deformation is measured at several locations by displacement transducers.

The equipment for opening $1.50 \mathrm{~m}$ deep slots includes a cutting machine, with a $1.00 \mathrm{~m}$ diameter diamond disk saw mounted at the end of a rig that houses the system that transmits the rotating movement to the disk. A central $168 \mathrm{~mm}$ diameter hole with a depth of $1.10 \mathrm{~m}$ is previously drilled by the same machine, in order to allow the disk saw supporting column to cut the slot down to the desired depth. Once the slot is cut, a flat jack is introduced and the central hole is filled with mortar (Figueiredo et al. 2010).

Each flat jack consists of two steel sheets, less than $1 \mathrm{~mm}$ thick, welded around the edges (Fig. 1a). Inside the flat jack there are four transducers for measuring the opening of the slots (Fig. 1b). Each transducer consists of two small steel cantilevers with four electric strain gauges. They are calibrated before being placed inside the jack in contact with both sides of the jack. Displacements are also measured at the surface of the rock mass. Previously to slot cutting, two pairs of measuring pins are placed $100 \mathrm{~mm}$ to each side of the slot and $175 \mathrm{~mm}$ from the jack axis of symmetry (Fig. 1c).
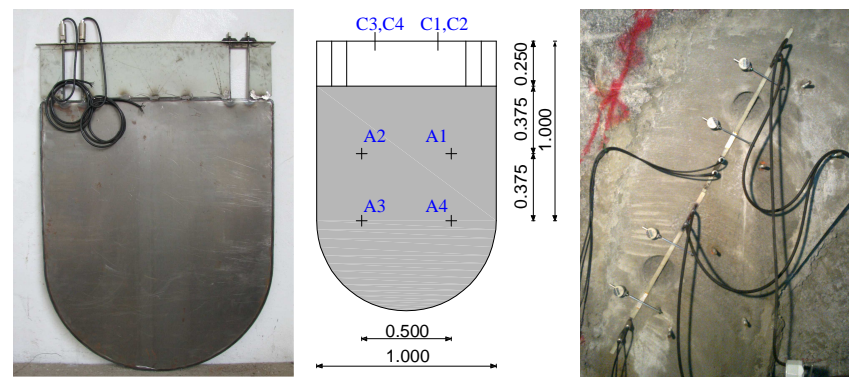

Figure 1. a) Large flat jack; b) location of the displacement transducers; c) ready for testing jacks in two contiguous slots.

The flat jacks are initially filled with oil and a low initial pressure, usually of about $0.05 \mathrm{MPa}$, is applied, in order to guarantee that the jacks are in contact with the slot wall. A LFJ test consists of, at least, three loading and unloading cycles with increasing maximum pressures. The raw test results are the pressure versus displacement curves obtained in the test.

In order to obtain a mean value of the modulus of deformability in large rock volumes, as well as information about the rock mass heterogeneity, two co-planar contiguous slots are usually opened for each test. 
Interpretation of flat jack test results is not straightforward, due to the development of a tensile crack along the plane of the slots as applied pressures increase. Crack initiation and propagation depends not only on applied pressure but also on the in situ stresses and rock mass tensile strength, which are generally unknown parameters (Pinto 1981, ISRM 1986).

To deal with this difficulty, a three-dimensional numerical model was recently developed for the interpretation of the large flat jack test results, enabling to simulate several cases of test geometries with two co-planar contiguous slots. The model allows simulating an actual large flat jack test protocol with several loading and unloading cycles (Figueiredo et al. 2011), and can also be used for estimating the initial in situ stresses perpendicular to the slots (Figueiredo et al. 2010).

Applications of the model to several cases are presented in this paper. They consider different cases regarding the relation between the initial stress normal to the slots and the rock mass tensile strength, the applied pressures, the loading and unloading cycles, or the loaded area (one or two loaded flat jacks). The influence of the tension crack on the test results is assessed and discussed.

\section{NUMERICAL MODEL}

For the interpretation of LFJ tests, a three dimensional finite differences model using the software FLAC3D (Itasca 2009) was developed. It can simulate a LFJ test, with two co-planar contiguous slots, performed at an angle between the plane of the slot and the rock mass surface (Figueiredo et al. 2010). The mesh is a $30 \mathrm{~m} \times 30 \mathrm{~m} \times 15 \mathrm{~m}$ solid and has 149,440 zones.

In order to simulate with detail the crack initiation and propagation into the rock mass, a very refined mesh around the slots was required, since they are just a few millimetres thick. Figure 2 (top left) shows a view of the mesh, and Figure 2 (top right) displays a middle vertical cross-section. The dark zones are the refined mesh, which is presented magnified at the bottom.

To simulate the tension crack, in the plane of the slots, interface elements with a finite tensile strength were used. Interface elements remain elastic for stresses below a given tensile strength; otherwise, a crack develops and the displacements versus pressure curves reveal a nonlinear behaviour.

The numerical model performs calculations that simulate both the cutting of the slots and the loading-unloading cycles. The initial stages comprise the following steps:

- installation of the in situ state of stress;

- completion of the central hole for the right side column;

- opening of the right side slot and filling of the central hole with mortar;

- completion of the central hole for the left side column;

- opening of the left side slot and filling of the central hole with mortar.

Afterwards, loading and unloading cycles can be modelled, considering all possible loading cases (Fig. 3):

- two loaded jacks in two contiguous slots;

- one loaded jack in two contiguous slots;

- a single flat jack in one slot.
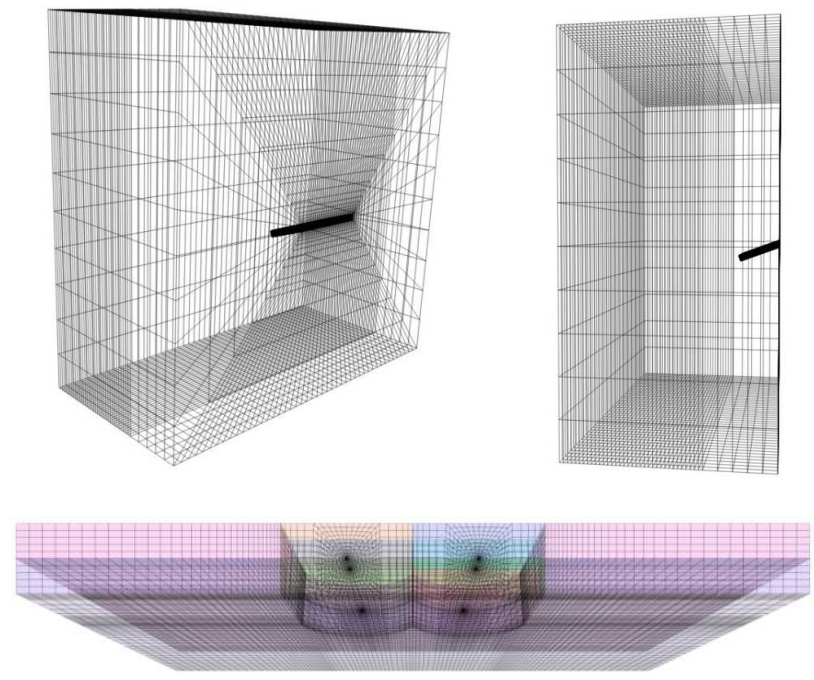

Figure 2. Three dimensional finite difference model.
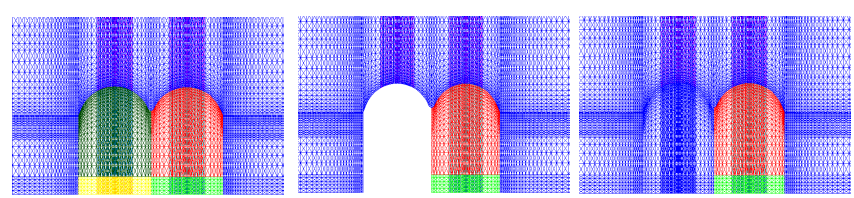

Figure 3. Interface for typical cases in LFJ tests: A - two slots and two loaded flat jacks; $\mathrm{B}$ - two slots and one loaded flat jack; $\mathrm{C}-$ one slot and one loaded flat jack.

The model allows assessing the depth of the crack $h$ for each loading step, and the evolution of the displacements in the rock mass, namely at the location of the transducers and along the alignments presented in Figure 4.

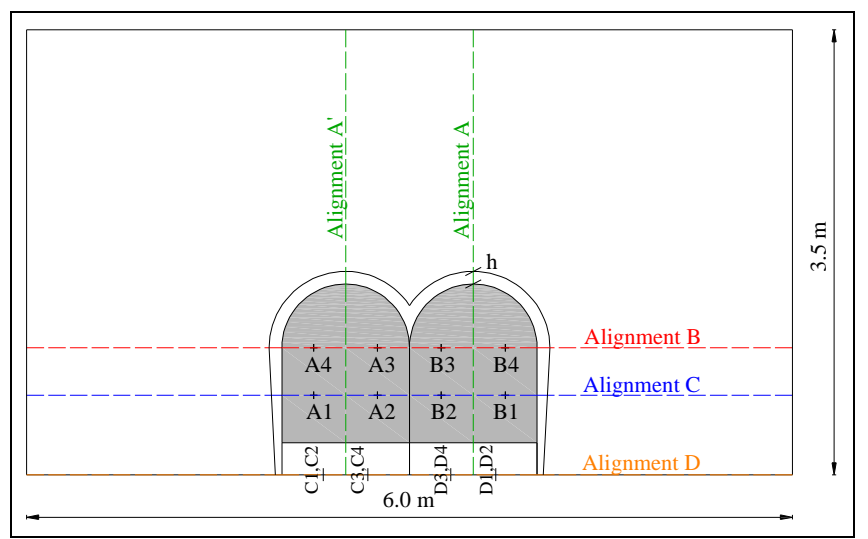

Figure 4. Location of the measuring points and alignments.

\section{CALCULATION OF THE TENSILE CRACK DEPTH}

\subsection{General}

Pressures applied by the jacks to the slot walls often induce tensile stresses along the edges of the slots that exceed the tensile strength of the rock mass, and therefore a crack starts to develop on the plane of the slots. Since determination of the rock mass modulus of deformability depends on the 
extent of the crack at each test stage, interpretation of the test results can be problematic due to the lack of information regarding the crack extent, which is difficult to evaluate even if the crack is visible at the rock mass surface.

Besides the applied pressure, two other parameters influence the development of the crack during a LFJ test: the tensile strength of the rock mass $\sigma_{t}$, and the initial in situ stress component perpendicular to the plane of the slots $\sigma_{z z}$. The former is a rock mass property, which depends on the rock tensile strength and on the jointing pattern. In good quality rock masses, without important joints, it can be nearly equal to the tensile strength of the rock, whereas in weak and fractured rock masses its value is so low that it can be neglected.

\subsection{Influence of the in situ stresses and tensile strength on the tensile crack depth}

In this section, following an approach by Pinto (1983), the relations between crack depth $h$, applied pressure $p$, in situ stress $\sigma_{z z}$, and tensile strength $\sigma_{t}$ are presented. Considering a rock mass with an isotropic, elastic and linear behaviour, the tensile stress $\sigma_{l}$ at the tip of the crack resulting from the application of a pressure $p$ by the flat jacks may be written as a function $f(h)$ of the tensile crack depth $h$ :

$\sigma_{1}=f(h) p$

Likewise, the compressive stresses $\sigma_{2}$ at the edge of the crack for a null applied pressure by the flat jacks may be written as a function $g(h)$ of the tensile crack depth:

$\sigma_{2}=g(h) \sigma_{z z}$

The crack reaches a given depth $h$ when the tensile stress $\sigma_{l}$ due to the application of the pressure $p$ is equal to the sum of the compressive stress $\sigma_{2}$ and the tensile strength of the rock mass $\sigma_{t}$ :

$\sigma_{1}=\sigma_{2}+\sigma_{t}$

Substituting equations (1) and (2) in equation (3), and dividing both sides by $f(h)$, the following equation is reached:

$p=\frac{g(h)}{f(h)} \sigma_{z z}+\frac{1}{f(h)} \sigma_{t}=\psi(h) \sigma_{z z}+\varphi(h) \sigma_{t}$

Finally, a simple relation between $p, \sigma_{z z}$ and $\sigma_{t}$ is reached:

$\frac{p}{\sigma_{z z}}=\psi(h)+\varphi(h) \frac{\sigma_{t}}{\sigma_{z z}}$

Equation (5) shows that, for each value of the tensile crack depth, the ratio $p / \sigma_{z z}$ varies linearly with the ratio $\sigma_{t} / \sigma_{z z}$. It was here assumed that the functions $\psi(h)$ and $\varphi(h)$ are well represented by quadratic functions of $h$, given by:

$\psi(h)=a+b h+c h^{2}$

$\varphi(h)=d+e h+f h^{2}$
Thus, equation (5) can be written as:

$\frac{p}{\sigma_{z z}}=a+b h+c h^{2}+d \frac{\sigma_{t}}{\sigma_{z z}} h+e \frac{\sigma_{t}}{\sigma_{z z}} h+f \frac{\sigma_{t}}{\sigma_{z z}} h^{2}$

Equation (5) can also be used to determine the ratio $p / \sigma_{t}$. In this case, the equation of the three dimensional surface can be written as follows:

$$
\frac{p}{\sigma_{t}}=a \frac{\sigma_{z z}}{\sigma_{t}}+b \frac{\sigma_{z z}}{\sigma_{t}} h+c \frac{\sigma_{z z}}{\sigma_{t}} h^{2}+d+e h+f h^{2}
$$

Equation (8) is used for the particular and unlikely case when the in situ stress $\sigma_{z z}$ is null and equation (7) cannot be used.

\subsection{Calculation of the tensile crack depth}

In order to establish relations between the tensile crack depth and the ratios $\sigma_{t} / \sigma_{z z}$ and $p / \sigma_{z z}$, a parametric analysis involving a very large number of numerical calculations considering plausible values for the parameters was performed. The result of each calculation is a set of values $\left(h ; \sigma_{t} / \sigma_{z z} ; p / \sigma_{z z}\right)$ approximately complying with equation (7).

Five values of the tensile strength $\sigma_{t}$ were considered $(0$, $1,2,4$ and $8 \mathrm{MPa}$ ), the in situ stress was set as $1 \mathrm{MPa}$, and pressures $p$ between 0.5 and $32 \mathrm{MPa}$ were numerically applied to the slot walls. All calculations were performed using the numerical model presented above, and considering a Young's modulus of $1 \mathrm{GPa}$ and a Poisson's ratio of 0.2 .

Considering the most common case of LFJ tests (two loaded slots), each calculation rendered as final result a set of values $\left(h ; \sigma_{t} / \sigma_{z z} ; p / \sigma_{z z}\right)$. Linear multiple regression enabled to determine parameters $a$ to $f$ and to define the most probable three-dimensional surface relating crack depth and the ratios $\sigma_{t} / \sigma_{z z}$ and $p / \sigma_{z z}$. This surface is presented in Figure 5, which, given estimates of the tensile strength and of the in situ stress, allows to determine the tension crack depth.

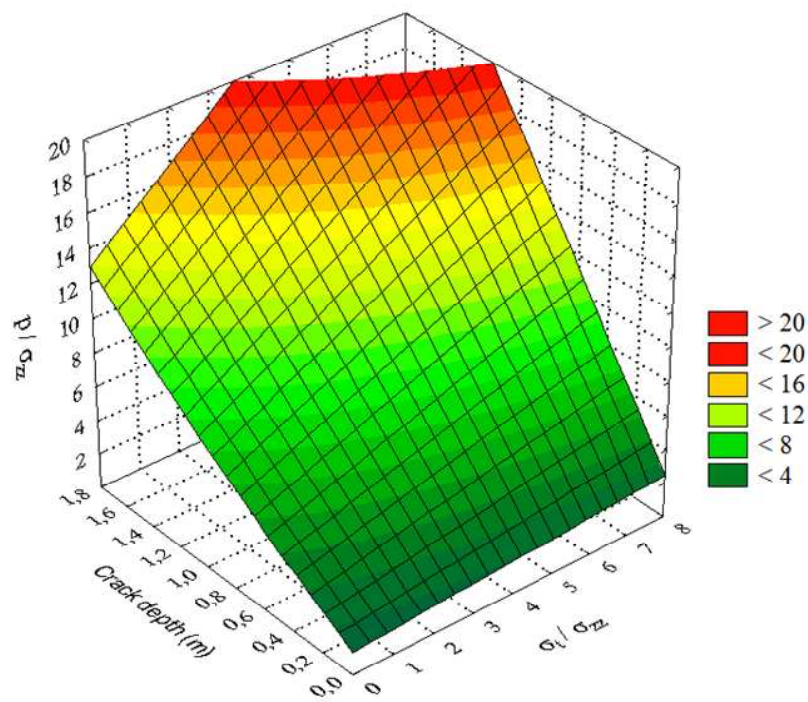

Figure 5. Three-dimensional surface relating crack depth with the ratios $p / \sigma_{z z}$ and $\sigma_{t} / \sigma_{z z}$ (two loaded slots case). 
Considering that one of the variables does not change, equation (7) can also be used to obtain the graphs relating the two remaining variables. So, the following graphs were created:

- relation between tensile crack depth and $p / \sigma_{z z}$ for several values of the ratio $\sigma_{t} / \sigma_{z z}$ (Fig. 6);

- variation of $p / \sigma_{z z}$ with $\sigma_{t} / \sigma_{z z}$ for several values of the tensile crack depth (Fig. 7);

- variation of $p / \sigma_{z z}$ with the crack depth for several values of the ratio $\sigma_{z z} / \sigma_{t}$ (Fig. 8).

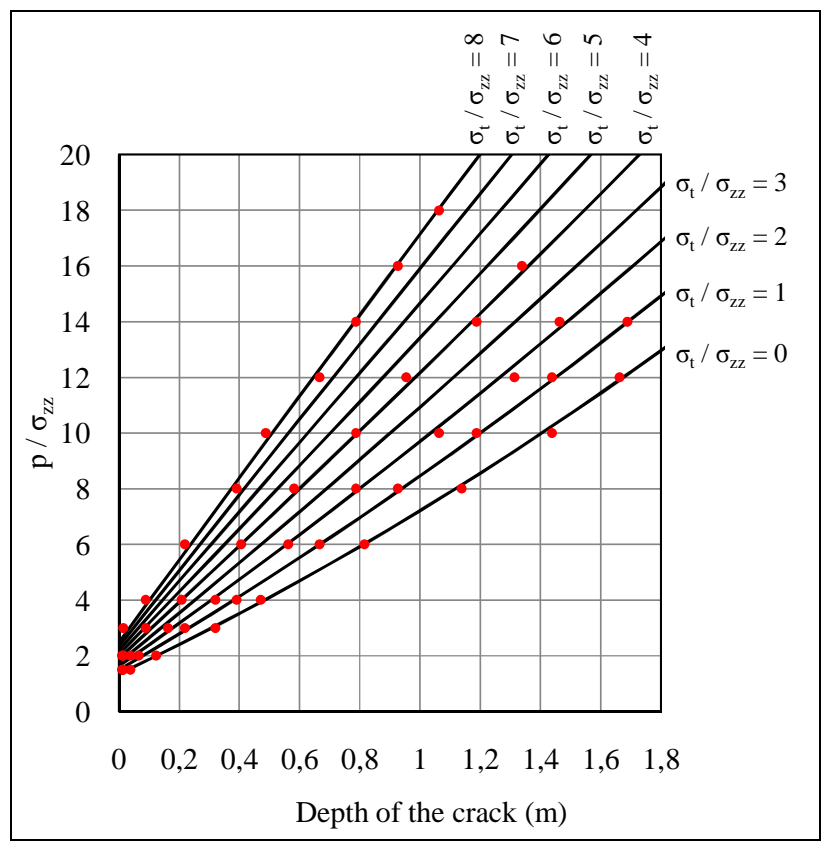

Figure 6. Variation of the ratio $p / \sigma_{z z}$ with the tensile crack depth for several values of the ratio $\sigma_{t} / \sigma_{z z}$.

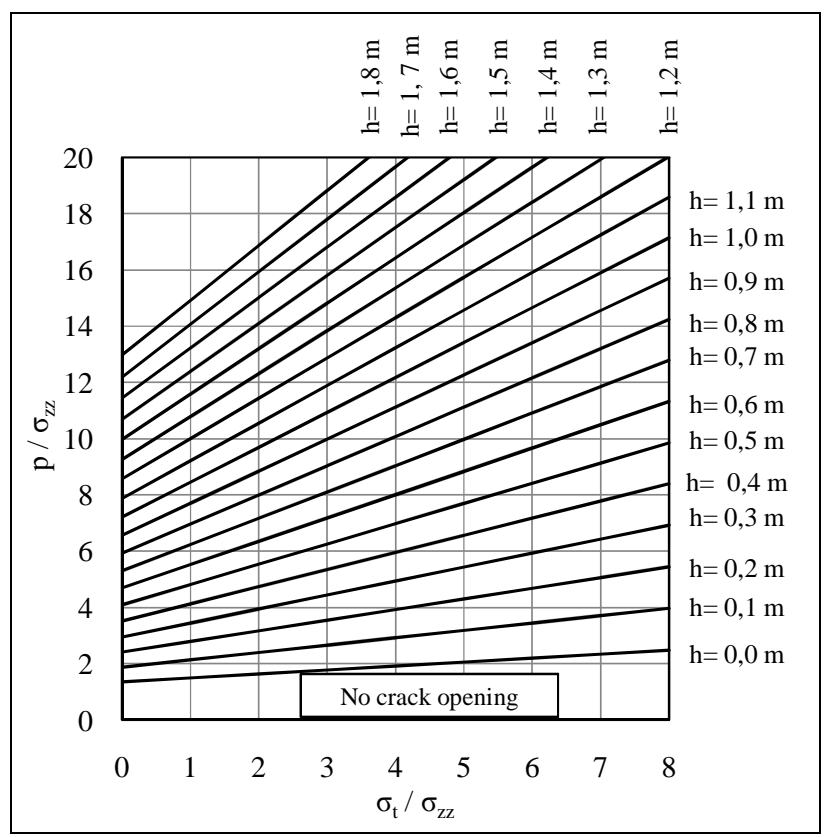

Figure 7. Variation of the ratio $p / \sigma_{z z}$ with the ratio $\sigma_{t} / \sigma_{z z}$ for several values of the tensile crack depth.

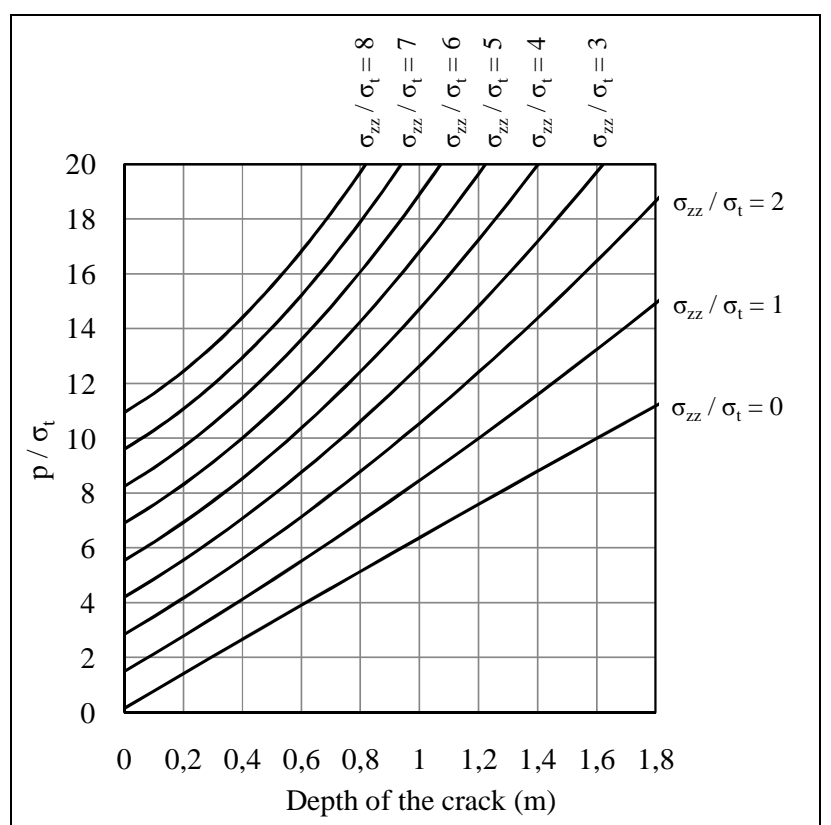

Figure 8. Variation of the ratio $p / \sigma_{t}$ with the tensile crack depth for several values of the ratio $\sigma_{z z} / \sigma_{t}$.

Dots displayed in Figure 6 correspond to some of the calculations performed with the numerical model. They show that a good approximation was reached.

As LFJ tests can be used to assess in situ stresses $\sigma_{z z}$ (Figueiredo et al. 2010) and tensile strength does not vary over a wide range of values, using Figure 7 it is possible to estimate crack depth propagation as pressure is applied during loading and unloading cycles of a LFJ test.

The same approach was followed to define similar relations for the remaining types of LFJ tests described in Figure 3: one loaded flat jack in two contiguous slots, and one loaded jack in a single slot.

Figure 9 presents graphs relating the ratio $p / \sigma_{z z}$ with the tensile crack depth for all the three analyzed cases. Figure 10 shows the normal stresses on the interface for $p / \sigma_{z z}=4 \mathrm{MPa}$ and for a null value of the rock mass tensile strength, allowing to appraise the extent of the tensile crack (in red) around the slots.
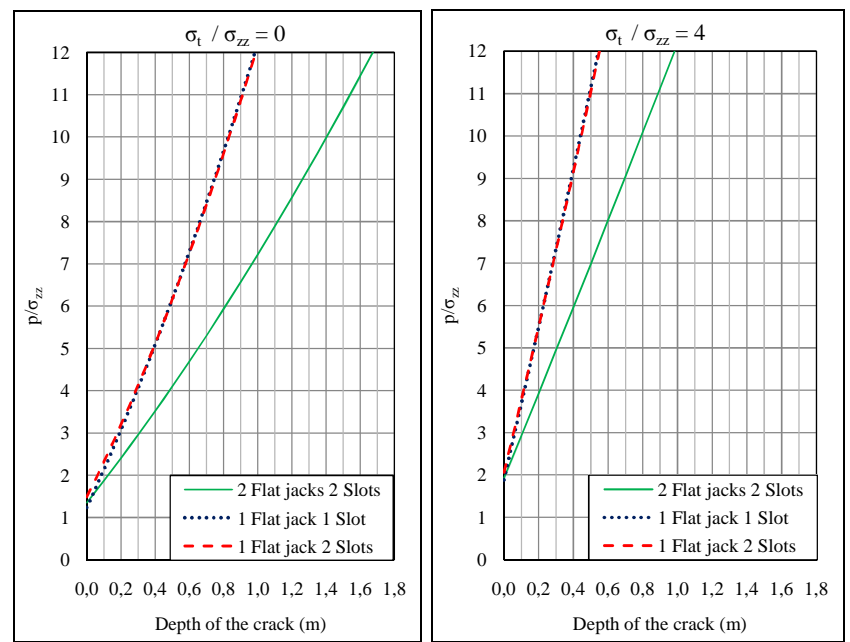

Figure 9. Variation of the tensile crack depth with the ratio $p / \sigma_{z z}$ for $\sigma_{t} / \sigma_{z z}=0$ and $\sigma_{t} / \sigma_{z z}=4$ 

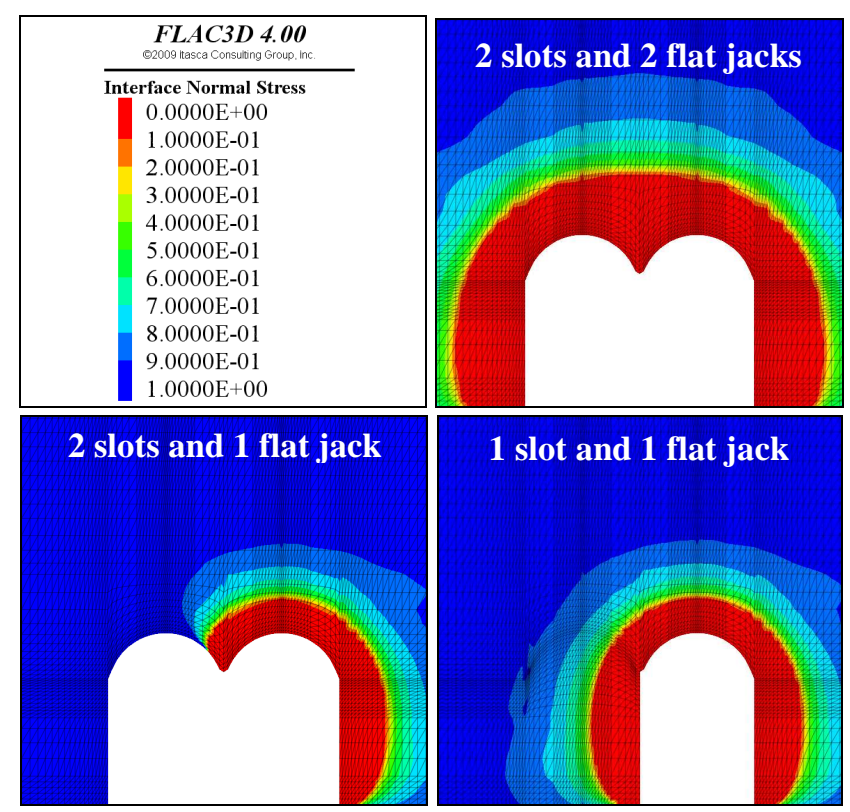

Figure 10. Normal stresses on the interface for $p / \sigma_{z z}=4 \mathrm{MPa}$ and $\sigma_{t} / \sigma_{z z}=0$.

\section{CALCULATION OF THE YOUNG'S MODULUS}

Several methodologies have been implemented in order to estimate the Young's modulus from the results of large flat jack tests. The model that has been used so far is based on the theory of elasticity for homogeneous, isotropic and elastic bodies and allows simulating a particular case, in which the slots are normal to the rock surface, and they are inserted in an infinite space from a rectangular testing chamber with a typical size: $3.5 \mathrm{~m}$ long and $2.5 \mathrm{~m}$ wide (Pinto 1983). The slot opening $\delta_{i}$ at measuring point $i$, corresponding to the variation of the pressure applied on the slot walls $\Delta p$, is given by:

$\delta_{i}=k_{i}\left(1-v^{2}\right) \frac{\Delta p}{E}$

where $E$ is the rock mass deformability modulus and $k_{i}$ is a coefficient depending on the number and combination of flat jacks, the location of the measuring point $i$, the shape of the test chamber and the tensile crack depth.

Coefficients $k_{i}$ may be used for interpretation of tests results independently of the rock mass mechanical parameters (modulus of deformability, Poisson's ratio, tensile strength, in situ stress). Pinto (1981, 1983) presents the values of $k_{i}$ for several combinations of loaded jacks and slots, given the tensile crack depth. Once having defined the values of $k_{i}$ at the location of each transducer, the most probable value of the deformability modulus $E$ of the rock mass can be obtained by minimising the sum of the squares of the differences between both sides of equation (9):

$E=\left(1-v^{2}\right) \Delta p \frac{\sum k_{i}^{2}}{\sum k_{i} \delta_{i}}$

The numerical model presented in this paper was used to calculate values of $k_{i}$ for a large number of situations, including several that couldn't be considered with the previous methodology. For each situation, considering a modulus of deformability of $1 \mathrm{GPa}$ and a Poisson's ratio equal to 0.2 , the numerical model was used for calculating, for each pressure increment, the tensile crack depth and the displacements at the locations where all displacement gauges are placed. Equation (9) is then used to obtain the values of the coefficients $k_{i}$ for each crack depth.

Figures 11, 12 and 13 show the diagrams with the variation of the coefficients $k_{i}$ with the tensile crack depth for the three loading cases presented in Figure 3. Table 1 presents the values of the coefficients $k_{i}$ obtained with the numerical model. These diagrams show that the coefficients increase as the tensile crack depth increases till a maximum value. For instance, in the case of two slots and two flat jacks, the diagram shows that the coefficients $k_{i}$ at the location of the transducers A2 and B2 are higher than the coefficients at the location of the others transducers. This may be explained since the transducers A2 and B2 are located closer to the rock mass surface in a lesser confined region and consequently the displacements are higher in these locations.

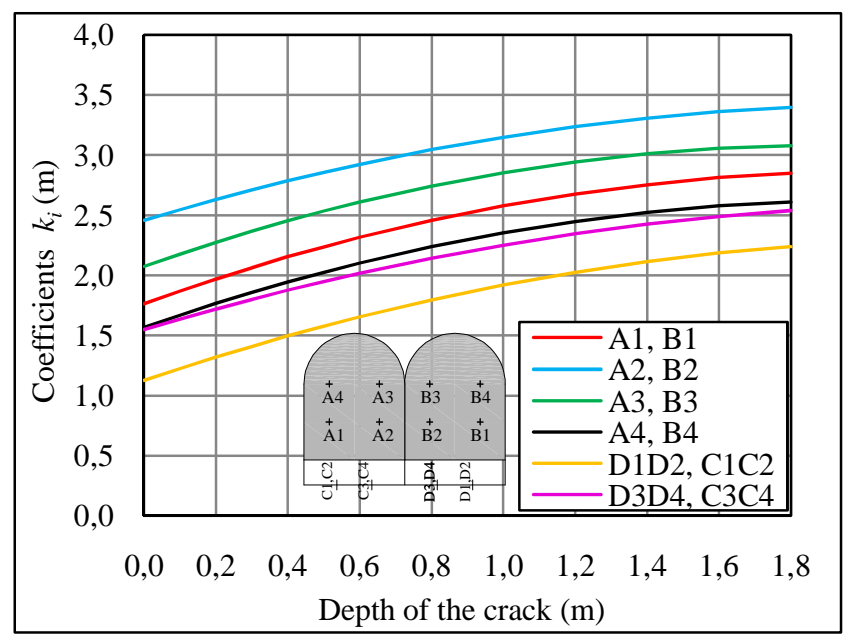

Figure 11. Variation of the coefficients $k_{i}$ with the tensile crack depth. Case A: two slots and two flat jacks.

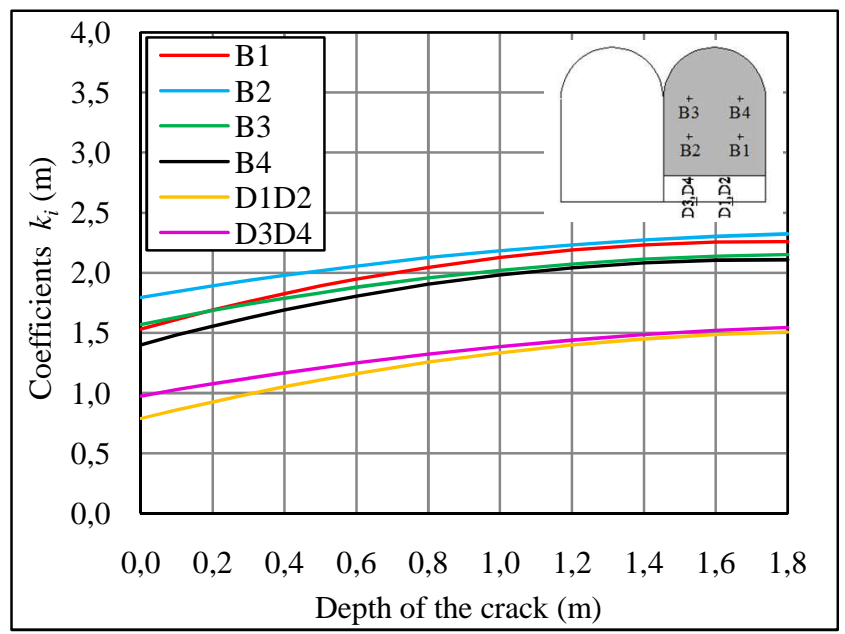

Figure 12. Variation of the coefficients $k_{i}$ with the tensile crack depth. Case B: two slots and one flat jack. 


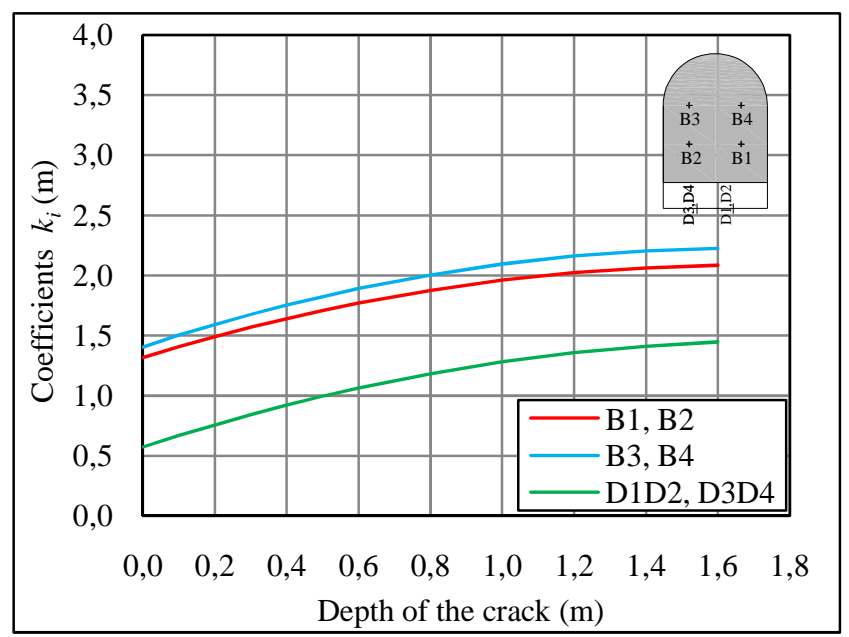

Figure 13. Variation of the coefficients $k_{i}$ with the tensile crack depth. Case $\mathrm{C}$ : one slot and one flat jack.

Table 1 . Coefficients $k_{i}$ computed by the numerical model.

\begin{tabular}{|c|c|c|c|c|c|c|}
\hline \multirow{2}{*}{ Case } & \multirow{2}{*}{$\begin{array}{c}\text { Gauge } \\
\text { location }\end{array}$} & \multicolumn{5}{|c|}{ Depth of the crack (m) } \\
\hline & & 0.0 & 0.2 & 0.5 & 1.0 & 1.6 \\
\hline \multirow{6}{*}{ A } & $\mathrm{A} 1, \mathrm{~B} 1$ & 1.764 & 1.969 & 2.237 & 2.577 & 2.811 \\
\hline & $\mathrm{A} 2, \mathrm{~B} 2$ & 2.458 & 2.630 & 2.856 & 3.149 & 3.362 \\
\hline & A3, B3 & 2.083 & 2.279 & 2.533 & 2.851 & 3.056 \\
\hline & $\mathrm{A} 4, \mathrm{~B} 4$ & 1.569 & 1.767 & 2.025 & 2.352 & 2.577 \\
\hline & $\mathrm{C} 1 \mathrm{C} 2, \mathrm{D} 1 \mathrm{D} 2$ & 1.115 & 1.313 & 1.576 & 1.920 & 2.182 \\
\hline & $\mathrm{C} 3 \mathrm{C} 4, \mathrm{D} 3 \mathrm{D} 4$ & 1.542 & 1.716 & 1.947 & 2.252 & 2.487 \\
\hline \multirow{3}{*}{ B } & $\mathrm{B} 1, \mathrm{~B} 2$ & 1.317 & 1.490 & 1.708 & 1.961 & 2.082 \\
\hline & B3, B4 & 1.406 & 1.590 & 1.824 & 2.094 & 2.225 \\
\hline & D1D2, D3D4 & 0.572 & 0.757 & 0.994 & 1.281 & 1.446 \\
\hline \multirow{6}{*}{$\mathrm{C}$} & $\mathrm{A} 1, \mathrm{~B} 1$ & 1.532 & 1.689 & 1.888 & 2.126 & 2.255 \\
\hline & $\mathrm{A} 2, \mathrm{~B} 2$ & 1.795 & 1.891 & 2.018 & 2.183 & 2.303 \\
\hline & A3, B3 & 1.570 & 1.686 & 1.835 & 2.020 & 2.138 \\
\hline & $\mathrm{A} 4, \mathrm{~B} 4$ & 1.401 & 1.555 & 1.750 & 1.982 & 2.105 \\
\hline & D1D2 & 0.787 & 0.926 & 1.107 & 1.335 & 1.486 \\
\hline & D3D4 & 0.975 & 1.076 & 1.210 & 1.386 & 1.519 \\
\hline
\end{tabular}

\section{DISCUSSION AND CONCLUSIONS}

Large flat jack tests were developed mainly for the study of concrete dams foundations. Its use decreased in the last decades due to the high costs of field tests, and also to the sometimes excessive use of classification systems and of empirical correlations that provide estimates of the rock mass deformability, based on a number of rock mass characteristics. However, for design of important structures, sensitive to rock mass deformation, after a preliminary investigation using indirect methods and empirical procedures, it is essential to obtain field values of the deformability from reliable tests. LFJ tests using two adjacent jacks are well suited to cost-effectively characterize rock mass deformability, since they are able to mobilize a relatively large volume of rock.

A difficulty in the interpretation of flat jack test results has to do with the tensile crack that may develop in the plane of the slots as the applied pressures increase. Crack initiation and propagation depend on the in situ stresses, rock mass tensile strength and applied pressure. Recently, a numerical model was developed for the interpretation of LFJ tests reproducing the exact geometry of the test and all test stages, as well as the development of the tension crack. This numerical model allows to estimate in situ stresses perpendicular to the slots and to model rock mass deformation during the loading and unloading cycles of the LFJ tests (Figueiredo et al., 2010 and 2011).

This paper presented a methodology for determining the tensile crack depth for several geometries and loading conditions, from the values of the applied pressure, the tensile strength and the initial stress normal to the jacks. It was demonstrated that, for each value of the tensile crack depth, the ratio $p / \sigma_{z z}$ varies linearly with the ratio $\sigma_{t} / \sigma_{z z}$ and, therefore, all calculations could be done as a function of these adimensional parameters. Values of the coeficients $k_{i}$ for calculation of the modulus of deformability were presented as a function of the tensile crack depth for several common geometries and loading conditions.

Studies are now being carried out that consider different situations as regards the inclination of the slots with respect to the rock face and different bounday conditions that simulate different test chamber geometries.

\section{ACKNOWLEDGEMENTS}

This work was carried out as part of LNEC's Programmed Research Plan, under the project "Determination of the geomechanical parameters of rock masses" and included research carried out for a MSc thesis presented by one of the authors to the New University of Lisbon.

\section{REFERENCES}

Bernardo, F. 2011. Numerical modelling of LFJ tests for the determination of rock mass deformability (in Portuguese). MSc thesis, New University of Lisbon.

Figueiredo, B., Lamas, L. \& Muralha, J. 2010. Determination of in situ stresses using large flat jack tests. ISRM International Symposium 2010 and $6^{\text {th }}$ Asian Rock Mechanics Symposium, New Delhi.

Figueiredo, B., Lamas, L. \& Muralha, J. 2011. Numerical simulation of a large flat jack test with cyclic loading. Proceedings of the $6^{\text {th }}$ International Conference on Dam Engineering, LNEC, Lisbon.

ISRM. 1986. Suggested method for deformability determination using a large flat jack technique. International Journal of Rock Mechanics Mining Sciences and Geomechanics Abstracts, 23: pp. 131-140.

Itasca. 2009. FLAC3D, Version 4.0, User's Manual. Itasca Consulting Group, Minneapolis, USA.

Pinto, L.J. 1981. Determination of the deformability modulus of weak rock masses by means of large flat jacks. Proceedings of an International Symposium on Weak Rock, Tokyo, pp. 447-452.

Rocha, M. 1974. Present possibilities of studying foundations of concrete dams. Proceedings of the 3rd ISRM International Congress on Rock Mechanics, Denver, USA, pp. 879-897.

Pinto, L.J. 1983 Deformability - LFJ Method (in Portuguese). In Recent developments in Rock Mechanics. LNEC, Lisbon. 